Recent Insights into the Physics of the Sun and Heliosphere:

Highlights from SOHO and Other Space Missions

IAU Symposium, Vol. 203, 2001

P. Brekke, B. Fleck, and J. B. Gurman eds.

\title{
Stellar Coronae
}

\author{
J. H. M. M. Schmitt \\ Hamburger Sternwarte, 21029 Hamburg, Gojenbergsweg 112, Germany
}

\begin{abstract}
.
The last two decades have seen the emergence of a new field in stellar astrophysics, the study of stellar coronae mostly in X-ray domain. With the advent of soft X-ray imagery X-ray emission was found from many thousands of solar-like stars. I will summarize the most important findings of X-ray surveys of late type stars and put the results in the context of the solar-stellar connection.
\end{abstract}

\section{Introduction}

A rather new view of the Sun opened when high-resolution telescopes operating at X-ray and extreme ultraviolet wavelengths became available to study the solar transition and corona. The high temperature of the outermost layers of the Sun orders of magnitude hotter than the solar photosphere - had been known since the 1940's, when the mysterious coronal lines seen in the optical were finally identified as forbidden emission lines from highly ionized iron. The solar X-ray images showed that the coronal emission was far from being anywhere close to spherically symmetric, rather a great number of loop-like structures filled with $\mathrm{X}$-ray emitting plasma were discovered. It became very obvious that magnetic fields had to play a key role both in structuring and heating the solar corona and hence one can view the solar corona as a prototype of a magnetically controlled thin plasma.

But what about stars? It is not possible to study stellar coronae in the optical waveband through the use of forbidden lines - any rather weak coronal emission would be overwhelmed by the strong stellar photospheric emission. Hot coronal plasma loses the bulk of its energy in the X-ray range, and thus not surprisingly, a systematic study of stellar coronae is only possible in the X-ray domain. In passing I do wish to point out the success of stellar radio observations, although one must keep in mind that radio emission from the quiescent Sun cannot be detected even from the nearest stars and therefore the detection of stellar radio emission is restricted to the class of active stars. The number of known X-ray sources has tremendously increased over the last two decades. I list the number of known coronal X-ray sources as a function of time in Tab. 1. With the end of the ROSAT operations we now know X-ray emission from far more than 20000 stars, and with Chandra and XMM operating hopefully throughout the next decade, this number will rapidly increase again. As is obvious from Tab.1, the breakthrough of stellar X-ray astronomy came 
with the advent of imaging soft X-ray telescopes, with the Einstein Observatory - operated between 1978 - 1981 - being the first such facility.

The Einstein Observatory demonstrated for the first time the ubiquity of stellar X-ray emission. At that time this was a big - since unexpected - surprise. But why should stellar X-ray emission be considered as a surprise, given that the Sun is a soft X-ray source? The surprise was not the fact that other stars are X-ray sources, but rather the surprise was their high level of X-ray emission. Given the Sun's soft X-ray luminosity of $\approx 2 \times 10^{27} \mathrm{erg} / \mathrm{sec}$ at solar maximum, it is trivial to compute the distance out to which solar-like X-ray emission can be detected at the sensitivity level of the ROSAT all-sky survey of $f_{X, l i m} \sim 2 \times 10^{-13} \mathrm{erg} / \mathrm{cm}^{2} / \mathrm{sec}$. The answer is $9 \mathrm{pc}$, i.e., a distance small compared to the dimensions of the Galaxy. A sphere of $9 \mathrm{pc}$ radius around the Sun does not contain 20000 stars and therefore the assumption that all stars emit $\mathrm{X}$-rays at the solar level must be incorrect. There must then exist stars with significantly higher activity levels which dominate the X-ray number counts. One then concludes that the Sun is by no means "typical" as far its activity and $\mathrm{X}$-ray emission is concerned and that surface temperature and total luminosity, the two parameters determining the position of a star in the HR-diagram, do not predict the level of activity and specifically the level of X-ray emission of a given star.

Solar physics can study the Sun in a detail that will never (?) be reached for stars, and the proceedings of this conference witness the remarkable achievements made in the observations and theoretical understanding of the Sun. Yet at the same time solar physics is limited by the uniqueness of the Sun. Solar physicists can only watch and observe what is happening on the Sun, they, however, cannot experiment with the Sun. But what would solar activity be like if the Sun were more massive or less massive, spinning faster or more slowly? What was the Sun like when it was young ? How active will it be in its old age? The answers to those and other questions are difficult to come by from studying only the Sun. With the large number of detected coronal X-ray sources we can in fact probe the dependence of stellar activity on stellar parameters like age, mass and rotation and thus by studying other stars the questions posed above can actually be addressed in a systematic way - and this is what the solar-stellar connection is all about.

Table 1. Coronal X-ray sources

\begin{tabular}{|r|r|r|}
\hline Year & Sun & 1 \\
\hline 1948 & Sun, Capella & 2 \\
\hline 1975 & RS CVn systems & 8 \\
\hline 1978 & ROSAT & $\approx 1500$ \\
\hline 1981 & Einstein Observatory & $>20000$ \\
\hline 1991 &
\end{tabular}




\section{What can be learnt from the Sun?}

The first and obvious question to ask is what one would be able to infer about the solar corona and - a bit more general - about solar activity at large if the Sun were placed at a distance of, say, $10 \mathrm{pc}$ and only "stellar" data, i.e. diskintegrated data with the currently achievable instrumental sensitivities, were at our disposal. The solar corona would not be detectable in optical forbidden lines, nor in the hard $\mathrm{X}$-ray or in the radio range; the coronal emission in these bands is either too weak or would be simply overwhelmed by the strong photospheric emission. Also, evidence for surface magnetic fields would be very hard to find. For Doppler imaging studies the Sun would rotate far too slowly, and the measurement of the flux changes when Sun spots cross the solar disk are so small that they would be detectable only from space. The only sign of magnetic activity from ground-based observations could be obtain from calcium data. Long-term studies of the line core fluxes would reveal both the rotation of the Sun and the cyclic activity pattern of solar activity. At soft X-ray wavelengths the Sun would appear as a rather weak, albeit clearly detectable, source. For an instrument like the ROSAT PSPC, which was the instrument used for the ROSAT all-sky survey, the solar X-ray emission would appear rather soft with most of the emission being at energies below $0.5 \mathrm{keV}$. The X-ray emission is also rather time variable, with variability being present on almost all time scales. Ignoring short term variability in the form of flares, the Sun's total Xray output undergoes significant changes on the cycle time scale of eleven years with variability amplitudes of a factor $10-100$ depending on the precise spectral range chosen. At any rate, the cyclic variability of the solar X-ray flux would become immediately obvious. The cyclic variability of the solar X-ray emission has been most impressively demonstrated with long-term synoptic series of $Y O H K O H$ images, which show the dramatic change of the appearance of the solar X-ray corona in the time between 1992 and 1996 between solar maximum and solar minimum. On shorter time scales such as the rotation time scale of one month, the observed variability amplitude is a factor $\approx 3$. Rotational modulation can often been seen in the Sun's total broad band X-ray emission in data covering similar spectral bands as the Einstein and ROSAT stellar X-ray data, but especially at higher energies rotation can masked by flaring variability. The observed solar X-ray emission never drops to zero, but remains at typically a third of fourth of the observed peak emission. In "stellar language" one would say that the same percentage of flux is not rotationally modulated, however, as is clear from the many solar images, it is not the case that this flux is located at high altitudes above the solar surface or near the poles. All types of solar variability, i.e., flares, rotational modulation and cyclic changes, could easily be detected even for a weak X-ray source like the Sun at typical stellar distances given sufficient data coverage; unfortunately, the life times of the satellites are usually much shorter than the activity cycle of the Sun and of other stars and there are no space-based facilities dedicated to monitoring only stars. Therefore long-term X-ray lightcurve of stars are very sparse and even the number of stars monitored in the calcium line is very small.

High resolution spectroscopic observations of the full solar disk similar to the stellar observations from the spectrometers onboard EUVE and now onboard Chandra and XMM, have been available in a solar context for more than twenty 
years ago (cf., Malinovsky \& Heroux 1973). The same methods of analysis that were applied to these solar data can now be applied to the new stellar data. In this fashion information on stellar coronal temperatures, emission measures, coronal chemical abundances, coronal densities and some structural information can be obtained.

\section{Stellar coronae across the HR-diagram}

Our knowledge of coronal X-ray emission has been derived almost exclusively from observations carried out with the Einstein Observatory, (operated between 1978 and 1981) and ROSAT (operated between 1990 and 1998); coronal X-ray sources were also intensely studied at extreme ultraviolet wavelengths with the EUVE satellite operated between 1992 and 2000. Stars were of course observed also with other X-ray satellites such as EXOSAT, ASCA and BeppoSAX, but the contributions from those missions were geared to more specific topics. Considerable impact on stellar X-ray astronomy is of course expected from the now operating Chandra and XMM-Newton observatories, but it is far too early to actually assess this impact. I consider the important findings in the field of stellar X-ray astronomy as follows:

1. The discovery of hot, X-ray emitting coronae on almost all types of stars with outer convection located on the main sequence.

2. The discovery of a dividing line in the H-R diagram separating stars evolving away from the main sequence into two groups, i.e., stars with hot coronae and stars with cool winds.

3. The discovery of relations between activity (as measured in terms of X-ray emission) and the rotation of stars.

4. The discovery of relations between activity (as measured in terms of X-ray emission) and the rotation of stars.

5. The discovery of X-ray flares on other stars.

6. The discovery of structure in the coronae around other stars.

In this paper I will focus on items (1),(2) and (5). A review of ROSAT observations flares has been given by Schmitt (1994).

\subsection{X-ray emission from late type dwarf stars}

Observations with the Einstein Observatory had already demonstrated the ubiquitous occurrence of X-ray emission from main sequence stars with outer convection zones. In particular it appeared that that onset of outer convection zones as predicted by stellar structure theory is reflected by a rather vigorous onset of activity. This issue was first addressed observationally by Schmitt et al. (1985), who found an enormous increase in detection rate when going from stars with $B-V \sim 0.2$ to stars with $B-V \sim 0.5$. With ROSAT Schmitt (1997) and Schmitt et al. (1995) performed a systematic volume-limited study of all known 


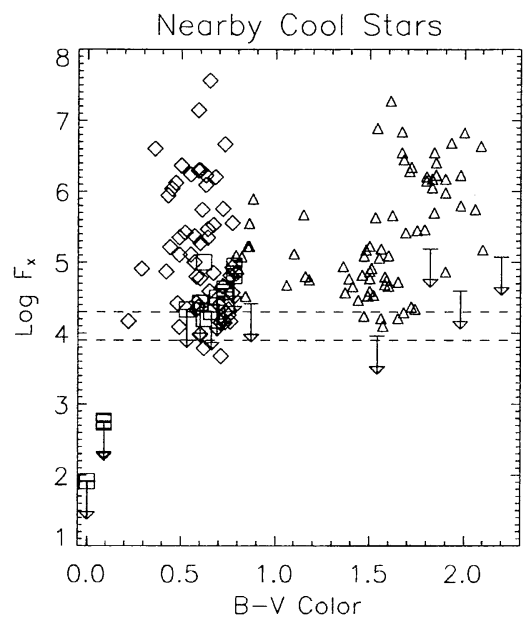

Figure 1. Mean X-ray surface brightness $F_{X}$ vs. $B-V$ color for my sample stars (including A-type stars drawn as upper limits). F and $G$ type stars are plotted with diamonds, $\mathrm{K}$ and $\mathrm{M}$ type stars (as discussed by Schmitt et al. 1995 with upward triangles. For comparison the typical X-ray surface flux level (in the PSPC band pass) from solar coronal holes is shown by the two dashed curves. Clearly the observed solar coronal hole surface flux provides a good description of the observed stellar minimum X-ray flux.

solar-like $\mathrm{F}$ and $\mathrm{G}$ type dwarf stars $(\mathrm{d}<13 \mathrm{pc}$ ) and $\mathrm{K}$ and $\mathrm{M}$ dwarf stars (d $<7 \mathrm{pc}$ ) using the ROSAT all-sky survey and pointed follow-up data (for stars not detected in the survey data). The basic result of this study is plotted in Fig. 1 as X-ray surface flux $F_{X}$ vs. B-V color for the solar-like stars studied by Schmitt (1997) as well as the $\mathrm{K}$ and $\mathrm{M}$ stars within $7 \mathrm{pc}$ studied by Schmitt et al. (1995). None of the three A-type stars in the immediate solar vicinity could be detected as X-ray sources, but for stars cooler than $\approx 8000 \mathrm{~K}$, the full range of X-ray activity is observed. The now available complete sample allows one to ascertain the question whether really all solar-like stars do possess coronae since the new sample is truly complete in the sense that all stars known within a distance of $13 \mathrm{pc}$ from the Sun were observed with sufficient to detect solar-like $\mathrm{X}$-ray emission levels (and even below). The detection rate among the F-type stars is very large, the hottest and among the F-type stars only non-detection being the star Gl 364 classified as F9 IV. The detection rate among the G-type stars exceeds $80 \%$, with all non-detections resulting from the lower sensitivity ROSAT survey data.

The same feature is observed if one looks at the X-ray detection rate among a stellar sample selected by optical magnitude. In a plot of X-ray detection rate vs. observed B-V color for stars of luminosity class V and/or IV contained in the Bright Star Catalog the detection rate shows a large increase at a color of B-V $\sim 0.3$ corresponding to spectral type F0 similar to the observed increase in the volume limited sample. Considering all the evidence one must therefore conclude 


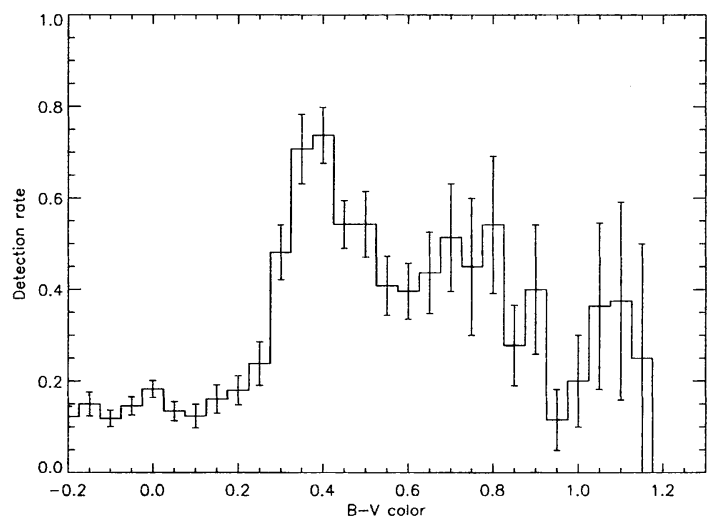

Figure 2. ROSAT all-sky survey detection rate for bright stars $\left(m_{v}<\right.$ 6.5) of luminosity class $\mathrm{V}$ and IV as a function of the observed $\mathrm{B}-\mathrm{V}$ color. Note the constant detection rates among hot stars and the rapid increase in detection rate at a $\mathrm{B}-\mathrm{V}$ color of $\mathrm{B}-\mathrm{V}=0.3$. Also note that if the same plot was produced with spectral type, the high detection rate among O-type stars would show up.

that coronal formation on solar-like stars represents an ubiquitous phenomenon. A corona containing hot plasma $\left(\mathrm{T}>10^{6} \mathrm{~K}\right)$ is always formed at the interface between a turbulent outer convection zone and space, and X-ray dark solar-like stars do not exist (at least within the immediate solar environment).

It is interesting to compare the mean X-ray surface fluxes for all stars of spectral type $\mathrm{F}$ through $\mathrm{M}$ (cf., Fig. 1). As obvious from Fig. 1, one observes a large spread in activity over $\approx 4$ orders of magnitude essentially independent of spectral type. However, one also notices in Fig. 1 the existence of a rather well defined lower envelope $F_{\text {lim }}$ to the observed mean X-ray surface flux distribution. The apparent cutoff at surface fluxes of $F_{\text {lim }} \approx 10^{4} \mathrm{erg} / \mathrm{cm}^{2} / \mathrm{sec}$ is not a question of lacking sensitivity since the non-detected A-type stars do indeed have upper limits below $F_{\text {lim }}$. Because of the samples' completeness properties both for the $\mathrm{F}$ and $\mathrm{G}$ stars as well as the $\mathrm{K}$ and $\mathrm{M}$ stars, one can state that among cool dwarfs stars with X-ray surface fluxes below $F_{\text {lim }}$ do not exist (in the considered volumes of space). The lower limit to the X-ray surface flux compares with the observed X-ray surface flux from solar coronal holes, which is indicated by the horizontal lines in Fig. 1. It is suggestive to interpret the stars observed at their minimum flux levels as stars surrounded by coronal holes without any active regions.

\section{Late M dwarfs and brown dwarfs}

According to the common paradigm of stellar activity one needs both convection and rotation - which are both needed for the operation of a dynamo - as necessary ingredients for the observed plethora of activity phenomena. Just as the onset of convection for late A/early F stars is of importance for dynamo theory, 
so is the very bottom of the main sequence. According to the stellar structure theory stars of spectral type dM5 or later, or in terms of mass, stars $\leq 0.3 M_{\odot}$, are expected to be fully convective, and should therefore not have a radiativeconvective interface. Such an interface is often employed by dynamo models to provide a place for magnetic flux storage, which otherwise would very quickly escape to the stellar surface because of the combined effects of convection and magnetic buoyancy. It is therefore an interesting result that the observed X-ray properties of M-dwarfs do not change for such fully convective dwarfs. Considering the ratio $L_{X} / L_{b o l}$, i.e., the efficiency, with which convective flux is eventually converted into X-ray flux, one does not find any evidence for any change neither for very low mass stars (Fleming et al. 1993), nor for field stars (Schmitt et al. 1995). While it is true that one of the latest known star, LHS 2924, could not be detected by Fleming et al. (1993), as well as BRI 0021-0214 (Neuhäuser et al. 1999), other rather similar stars have been detected (i.e., LHS 3003, cf. Schmitt et al. 1995), suggesting that the apparent lack of detections of very low mass stars (cf., Fleming et al. 1993) is due only to insufficient sensitivity given the the rather large distance of these objects (for LHS 2924 one finds an upper limit of only $L_{x} / L_{b o l}<4.510^{-5}$ ), and that X-ray emission probably occurs right to the bottom of the main sequence. Interestingly, X-ray emission has even been detected for a few brown dwarfs, i.e., objects below the hydrogen burning limit (at $\approx 0.08 \mathrm{M}_{\odot}$ ), but the small number of hitherto detected brown dwarfs prevents any far reaching conclusions. Interestingly, (some) very low mass stars and/or brown dwarfs seem to be able to produce at least rather massive transient energy releases. As an example we consider the ROSAT HRI light curve of the very low mass star vB10, reproduced in Fig. 3. During this $20 \mathrm{ksec}$ exposure (presented and analyzed in detail by Fleming, Giampapa and Schmitt 2000) no statistically significant detection could be obtained except for one observation interval of 20 minutes when a total of 11 (!) counts were recorded; at the distance of vB10, the observed HRI count rate translates into an X-ray luminosity of more than $2 \times 10^{27} \mathrm{erg} / \mathrm{sec}$ at flare peak. This has to be contrasted with the upper limit of $\sim 2 \times 10^{25} \mathrm{erg} / \mathrm{sec}$ for any quiescent X-ray emission from vB10. A similar event with lower peak X-ray luminosity and a lower upper limit to any quiescent X-ray emission was observed by Chandra on the brown dwarf LP 944-20 by Rutledge et al. (2000). From these few - admittedly still sparse - observations we tentatively conclude that magnetic activity can also be present among very low mass stars and that for the X-ray properties of a main-sequence stars it appears to be irrelevant whether the star's interior is fully convective or not.

\section{X-ray emission from giants and supergiants}

Energy transport through convection takes place not only for main sequence stars of spectral later than $\approx \mathrm{F} 7$, but also for giant stars. On the other hand, giant stars tend to be older than their corresponding main sequence cousins - so what about activity among giants ? The existence of an X-ray dividing line (XDL) among giant stars was first established by the Einstein Observatory. Hot coronae with temperatures $T \sim 10^{6} \mathrm{~K}$ or higher were found precisely for those stars where UV spectra obtained with the IUE satellite had indicated the presence of material at transition region temperatures (through, for example, 


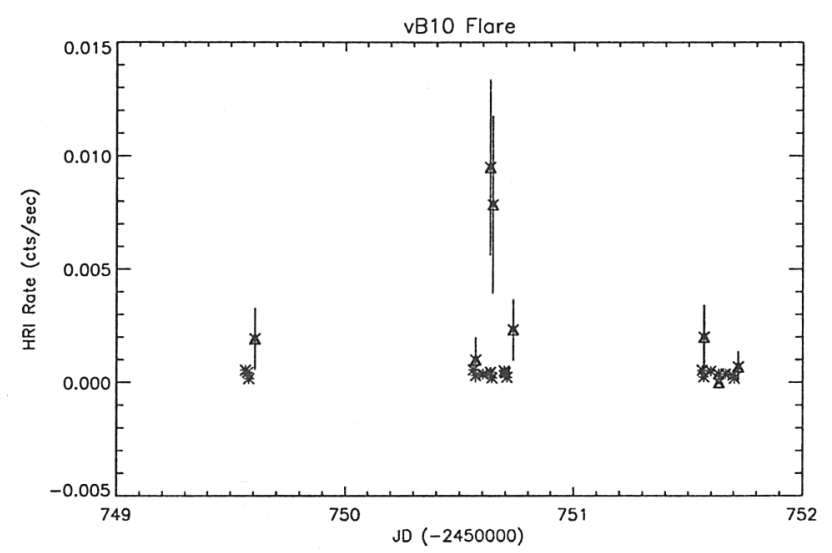

Figure 3. X-ray light curved observed with the ROSAT HRI for the very low mass star vB10. Over most of the observation interval no significant detection could be obtained. In a 20 minute interval during the course of the observations a significant detection has been made. The few photons do not allow the construction of a proper X-ray light curve, they are consistent however with the interpretation of a flare decay. The peak X-ray luminosity corresponds to levels of the quest Sun at solar maximum conditions.

CIV emission), while stars without such transition region emission were also devoid of coronal soft X-ray emission (cf., Linsky and Haisch 1979; Ayres et al. 1981), and instead indicate the presence of cool, massive winds. The existence of the dividing line has been impressively confirmed by the extensive material of the ROSAT All Sky Survey (cf., Haisch, Schmitt \& Rosso 1991; Haisch, Schmitt \& Fabian 1992) as well as the ROSAT pointing program (cf., Hünsch et al. 1996). Hünsch et al. (1997a) have produced a final catalog of detections of giants and supergiants listed in the BSC and detected in the RASS data; for 3839 such stars they find a total of 450 positional matches. Hünsch and collaborators have studied the few stars in seeming conflict with the dividing line (Haisch, Schmitt \& Rosso 1991, Hünsch et al. 1995, Hünsch et al. 1997b) and confirm the concept of an X-ray dividing line (XDL).

Just like on the main-sequence, X-ray emission for giants to the left of the dividing line seems to be ubiquitous. Hünsch et al. (1996) carried out a study of a complete volume-limited sample of giants within $25 \mathrm{pc}$ around the Sun using both ROSAT survey and pointing data. While the overall detection rate was only $50 \%$ for stars to the left of the DL, all stars observed in the pointing program with sufficient sensitivity to detect solar-like emission levels were in fact detected, a finding, which led Hünsch et al. (1996) to the conclusion that giants to the left of the DL, which all have outer convection zones, are also ubiquitous $\mathrm{X}$-ray emitters. For giants the DL occurs across a very narrow region in the $\mathrm{H}-\mathrm{R}$ diagram. Thus a $\mathrm{G}$ giant (to the left of the dividing line) can have a rather high X-ray luminosity of up to $L_{X} \sim 3 \times 10^{30} \mathrm{ergs} \mathrm{s}^{-1}$, although such objects seem to be relatively rare while $\mathrm{K}$ giants to the right of the dividing line can 
be almost five orders of magnitude fainter. For example, Ayres, Fleming and Schmitt (1991) were not able to detect the nearby giant Arcturus and obtained an upper limit of $L_{X}<3 \times 10^{25} \mathrm{ergs} \mathrm{s}^{-1}$; expressing this upper limit in terms of mean X-ray surface flux, this X-ray non-detection is more than 1000 times fainter than a solar coronal hole. As the visually brightest and nearest $\mathrm{K}$ giant, Arcturus is the best non-coronal star to study, however, it may not be "typical". It does appear to have some C IV emission (Ayres et al. 1995) with $f_{C I V} / f_{\text {bol }}$ more than an order of magnitude weaker than the Sun, but it must be rather old and of rather low mass.

The concept of a dividing line seems to disappear, however, among the brighter giants and supergiants. Among those stars there is a group of stars exhibiting both signatures of transition region material (as inferred from CIV line detections) as well as cool winds (inferred from UV line profiles), i.e., the so-called hybrid stars. When these objects were first proposed as a new class by Hartmann, Dupree and Raymond (1980) and by Reimers (1982) it was unknown whether they actually possessed X-ray coronae. As a result of extensive ROSAT observations hybrid stars are now known to possess hot coronal plasma at temperatures in the $10^{6}-10^{7} \mathrm{~K}$ range (Haisch, Schmitt \& Rosso 1992; Reimers \& Schmitt 1992; Kashyap et al. 1994; Reimers et al. 1996). The observational situation is summarized in Fig. 4, where the stars reported by Hünsch et al. (1996) within $25 \mathrm{pc}$ around the Sun and the hybrid stars discussed by Reimers et al. (1996) are shown in an HR-diagram. The nearby giants detected as X-ray sources are plotted as dark gray circles, those not detected as X-ray sources as white circles, the X-ray detected hybrid stars are shown as black circles and the hybrid stars not detected as X-ray sources as light grey circles. As is obvious from Fig. 4, the XDL shows very clearly up for giants of luminosity class III, while most of the hybrid stars have been detected as X-ray sources, some of them having a spectral type which puts them well beyond the XDL for luminosity class II giants.

Stars with masses $\geq 1.3 M_{\odot}$ are not expected to develop dynamo activity while on the main sequence since they are either fully radiative or have core convection zones at best. Stars with spectral types later than about B1.5V do not appear to be bona fide X-ray sources (Berghöfer et al. 1997). Therefore there is no apparent mechanism to brake the often observed very rapid rotation of such stars. However, as the stars evolve off the main sequence to become giants, one expects dynamo-sustaining convective envelopes to develop. An onset of coronal emission and a "coronal gap" between the main sequence and the turn-on point for such intermediate mass stars are therefore expected to arise. Observational evidence for such an "onset of convection" among giants has yet to be produced.

\section{Coronal structure}

Given the enormous range of X-ray luminosities of solar-like stars, one wonders how those stars, which essentially look like the Sun, manage to produce orders of magnitude higher X-ray outputs. What, then, do stellar coronal structures look like ? If one assumes, going along with the solar analogy, that the X-ray emitting plasma is magnetically confined in magnetic loops, should one then expect simply more loops than typically visible on the solar surface, higher 


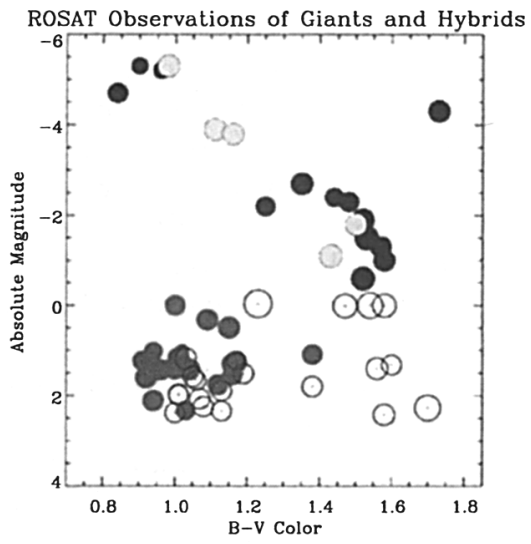

Figure 4. X-ray "bubblegram" of a complete sample of giants within 35 pc around the Sun (from Hünsch et al. 1996) and hybrid stars (discussed by Reimers et al. 1996). Plotted are the HR-diagram positions of nearby giants detected as X-ray sources (dark gray circles), the positions of nearby giants not detected as X-ray sources (white circles), the positions of hybrid stars detected as X-ray sources (black circles) and those of hybrid stars not detected as X-ray sources (light grey circles).

density loops or longer loops in order to account for the observed values of $E M$ and $L_{\mathrm{X}}$ ? Only solar observations have the luxury of spatial resolution, stellar coronae always appear as point sources. The classical method to address the question of stellar coronal structure are eclipse studies in suitably chosen systems where one tries to constrain the emitting plasma volume from the observed light curve. Starting with the eclipse studies of the eclipsing RS CVn system AR Lac (Walter, Gibson and Basri 1983), quite a number of such systems have been observed with the EXOSAT, ROSAT and EUVE satellites; a review of such observations has been given by Schmitt (1998). In most of the systems studies both components are late type stars and hence coronally active. Disentangling the light curve is an absolutely non-trivial task (cf., Schmitt 1996), and it is clear that for the purposes of eclipse mapping systems with one X-ray dark component, i.e., those containing an A-type or B-type star, are quite preferable. An extremely interesting system in this context is the nearby binary $\alpha \mathrm{CrB}$. The primary component is of spectral type A0V, the secondary component of spectral type G5V; the two stars are in an eccentric orbit $(\mathrm{e}=0.37)$ of 17.36 days. The system shows photometric eclipses, with primary eclipse (i.e., G star in front of A star) being annular, and secondary eclipse (i.e., A star in front of G star) being total; further, the system is a double lined spectroscopic binary. The radii of the binary components can be determined from light curve modeling as 3.0 and $0.9 R_{\odot}$ for $\mathrm{A}$ and $\mathrm{G}$ component respectively, and further, a full solution of the orbit motion (including the orbit inclination) can be obtained.

$\alpha \mathrm{CrB}$ is thought to be a member of the Ursa Major Stream, hence it should be relatively young ( $400 \mathrm{Myrs})$ and the G-star should exhibit activity at the Hyades level. These expectations were confirmed by the RASS detection of $\alpha$ 


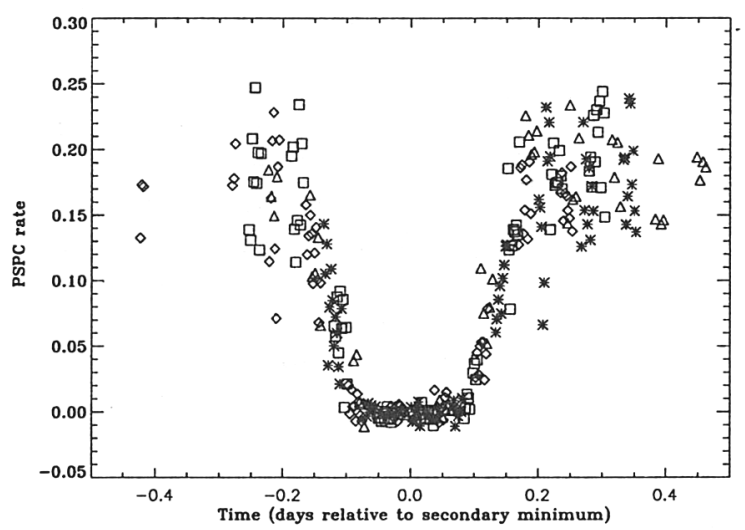

Figure 5. Overlay of all available ROSAT PSPC observations of $\alpha$ $\mathrm{CrB}$. A total eclipse is seen in all observations and consequently the length of X-ray totality is well defined.

CrB. At X-ray wavelengths one expects the A-type star to be dark, hence a total X-ray eclipse should occur at the time of optical secondary minimum. PSPC observations of the optical secondary eclipse were carried out on July 121992 , July 28-29 1993 , Aug 15-16 1993, and Feb. 19-20 1997. The first discovery observations were presented by Schmitt and Kürster (1993), the remaining data sets by Schmitt (1998). In Fig. 5 I show the superimposed light curves of all four observations; as is obvious from Fig. 5, total X-ray eclipses were observed in all three cases, and the total duration of the X-ray eclipse $t_{d u r, X-r a y}=0.356 \pm 0.027$ days agrees very well with the total duration of the optical eclipse $t_{d u r, o p t}=$ 0.35925 days. It therefore appears to be the case that the spatial extent of $\alpha \mathrm{CrB}$ B's corona is more or less confined to its visible surface, or putting it differently, the height of the corona above the limb appears to be considerably less than a stellar radius. I emphasize that this conclusion is independent from any modeling assumptions. One can of course go further and try to reconstruct the spatial distribution of the X-ray emitting plasma from the observed ingres and egress of the X-ray light curve. Naturally, this is an ill-posed problem since one is trying to reconstruct a two-dimensional distribution from a one-dimensional light curve and the resulting images have to be interpreted with care (cf., Schmitt 1996). At any rate, it is very clear that the images are inconsistent with a uniform plasma distribution, rather the X-ray emission must be inhomogeneous, probably concentrated in active regions similar to the solar X-ray emission.

\section{Summary}

Hopefully this presentation has demonstrated the enormous progress made in the study of stellar coronae. Compared to twenty years ago, the observational situation has dramatically changed with many thousands of coronal X-ray sources known. As a consequence it becomes less and less important to increase the number of known X-ray sources except for some very specific objects such as X- 
ray emitting brown dwarfs, X-ray emitting $\mathrm{F}$ type giants or X-ray sources with known age, rather the detailed study of individual sources through their light curves and X-ray spectra becomes more and more relevant. By necessity such observations are very time-consuming and therefore only a relatively small number of stars will be amenable to such detailed study. As far as X-ray spectroscopy is concerned, the recently launched Chandra and XMM-Newton observatories have the potential to acquire stellar X-ray data with (almost) the same spectral resolution as available for the solar corona and I expect that such studies will eventually lead to the clarification of fundamental physical parameters such as temperatures, densities and chemical abundances of stellar coronae.

\section{References}

Ayres, T.R., Linsky, J.L., Vaiana, G.S., Golub, L., \& Rosner, R., 1981, ApJ, 250,293

Ayres, T.R., Fleming, T.A., \& Schmitt,J.H.M.M., 1991, ApJL, 376, L45.

Ayres, T. R., Fleming, T. A., Simon, T., Haisch, B. M., Brown, A., Lenz, D., Wamsteker, W., de Martino, D., Gonzalez, C., Bonnell, J., Mas-Hesse, J. M., Rosso, C., Schmitt, J. H. M. M., Trümper, J., Voges, W., Pye, J., Dempsey, R. C., Linsky, J. L., Guinan, E. F., Harper, G. M., Jordan, C., Montesinos, B. M., Pagano, I. and Rodono, M., 1995, A\&AS , 96, 223

Berghöfer, T.W., Schmitt, J.H.M.M., Danner, R.,\& Cassinelli, J.P., 1997, A\&A Supp., 322, 167

Dupree A. K., Brickhouse N.S., Doschek G.A. et al. 1993, ApJL, 418, L41

Fleming, T.A., Giampapa, M.S., Schmitt, J.H.M.M. \& Bookbinder, J.A., 1993, ApJ, 410, 387.

Fleming, T. A.,Giampapa, M. S., Schmitt, J. H. M. M., ApJ, 2000, 533, 372.

Haisch, B., Schmitt, J.H.M.M. \& Rosso, C., 1991, ApJL, 383, L15.

Haisch, B., Schmitt, J.H.M.M. \& Rosso, C., 1992, ApJL, 388, L61.

Hartmann, L, Dupree, A.K., \& Raymond, J.C. 1980, ApJ, 236, L143

Hünsch, M., Reimers, D., and Schmitt, J. H. M. M., 1996, 313, 755

Hünsch, M., Schmitt, J. H. M. M., Schröder, K. -P. and Reimers, D., 1996, 310, 801

Hünsch, M., Schmitt, J.H.M.M. \& Voges, W.H., 1997, A\&A Supp, 127, 251

Kashyap, V., Rosner, R., Harnden, F.R. Jr., Maggio, A., Micela, G. \& Sciortino, S., 1994, ApJ, 431, 402.

Linsky, J.L. \& Haisch, B.M. 1979, ApJ, 229, L27

Neuhäuser, R., Briceńo, C., Comerón, F., Hearty, T., Martiacuten, E. L., Schmitt, J. H. M. M., Stelzer, B., Supper, R., Voges, W. and Zinnecker, H., A\&A, 1999, 343, 883

Neuhäuser, R. and Comerón, F., Science, 1998 Vol. 282, p. 83

Rutledge, R. E., Basri, G., Martín, E. L. and Bildsten, L., 2000, ApJ, 538 L141

Reimers, D., 1982, A\&A, 107, 292

Reimers, D. \& Schmitt, J.H.M.M., 1992, ApJL, 392, L55. 
Reimers, D., Hünsch, M., Schmitt, J.H.M.M. \& Toussaint, 1996, A\&A , 310, 801.

Schmitt, J.H.M.M., Golub, L., Harnden, F.R. Jr., Maxson, C.W., Rosner, R. and Vaiana, G.S., 1985, ApJ , $290,307$.

Schmitt, J.H.M.M. \& Kürster, M., 1993, Science, 262, 215.

Schmitt, J.H.M.M., 1994, ApJS, 90, 735.

Schmitt, J.H.M.M., Fleming, T.A. \& Giampapa, M.S., 1995, ApJ, 450, 392

Schmitt, J. H. M. M., 1996, IAU Symposium 176, ed. K. Straßmeier, 85

Schmitt, J.H.M.M., 1997, A\&A, 318, 215

Schmitt, J.H.M.M., 1998, A\&A, 333, 199

Schmitt, J. H. M. M., 1998, ASP Conf. Ser. 154: Tenth Cambridge Workshop on Cool Stars, Stellar Systems, and the Sun, 463

Walter, F. M., Gibson, D. M., and Basri, G. S. 1983, Ap.J., 267, 665. 